\section{Main Symposium}

MS-2 LI, Wen-Hsiung ${ }^{1}\left({ }^{1}\right.$ Department of Ecology and Evolution, University of Chicago)

Protein Function, Connectivity, Duplicability and Dispensability

Protein-protein interaction networks have evolved mainly through connectivity rewiring and gene duplication. However, how protein function affects these processes and how a network grows in time have not been well studied. Using protein-protein interaction data and genomic data from the budding yeast (Saccharomyces cerevisiae), we first examined whether there is a correlation between the age and connectivity of yeast proteins. A steady increase in connectivity with protein age is observed for yeast proteins except for those that can be traced back to eubacteria. Second, we investigated whether protein connectivity and duplicability vary with gene function. We found a higher average duplicability for proteins interacting with the external environments than for proteins localized within intracellular compartments. For example, proteins that function in the cell periphery (mainly transporters) show a high duplicability but are lowly connected. Conversely, proteins that function within the nucleus (e.g., transcription, RNA and DNA metabolisms, and ribosome biogenesis and assembly) are highly connected but have a low duplicability. Third, we found a negative correlation between protein connectivity and duplicability. Finally, we studied the effect of protein complexity on gene dispensability and duplicability.

MS-3 MATTICK, John S. ${ }^{1}$ ( ${ }^{1}$ Institute for Molecular Bioscience, University of Queensland, Brisbane QLD 4072, Australia)

The Hidden Layer of Noncoding Rna in the Genetic Programming of Complex Organisms

The current orthodoxy of molecular biology holds that genes are generally synonymous with proteins, and therefore that proteins not only fulfil the primary structural and functional roles within cells, but are also the main agents by which cellular dynamics are controlled, in conjunction with cis-regulatory elements and environmental signals. This is essentially true in prokaryotes, whose genomes are very largely comprised of closely spaced protein coding sequences, separated by 3' and 5' regulatory sequences that operate at the transcriptional and translational level.

It has been assumed that most genes also encode proteins in eukaryotes, despite the fact the proportion of protein-coding sequences broadly declines as a function of multicellularity and is only a small minority of the genomic programming of complex organisms like mammals. This assumption has led to several logical extensions and subsidiary assumptions, in particular that the majority of the non-protein-coding sequences in eukaryotic genomes is evolutionary debris (i.e. junk).

This may not be correct. Analysis of a variety of eukaryotic genomes indicates that the relative amount of non-protein-coding sequences and the amount of transcription of non-protein-coding RNA (noncoding RNA) increases as a function of developmental complexity. Recent evidence suggests that at least half of the genes in the mammalian genome do not encode proteins. Indeed, most of the mammalian genome is transcribed, the vast majority $(\sim 98 \%)$ of which is non-protein-coding RNA, comprising introns of protein-coding transcripts and introns and exons of non-proteincoding transcripts (ncRNAs). These transcripts include complex interlaced clusters of overlapping and antisense transcripts, "intergenic" transcripts and pseudogene transcripts that appear to participate in both local and long-distance regulatory networks. Many transcripts are processed to smaller RNAs, including snoRNAs that edit other RNAs, and microRNAs that control many aspects of development, including embryogenic patterning, adipocyte formation, hematopoietic differentiation, apoptosis and insulin secretion, and are perturbed in a range of cancers.

RNA signaling is also involved in chromosome dynamics and chromatin modification, epigenetic processes which, like alternative splicing (also likely to be controlled by trans-acting RNAs), are essential to differentiation and development. Interestingly many ncRNAs with conserved functions, such as XIST and H19, are not highly conserved at the sequence level, suggesting that (like language) their sequences can drift easily and yet retain function, which also suggests that there may be many more microRNAs and other regulatory RNAs to be discovered. Many putative ncRNAs identified in the RIKEN mouse cDNA project exhibit tissue-specific expression patterns and are dynamically induced by physiological stimuli. Interestingly ncRNA expression and RNA editing is particularly high in the brain. There are also many nucleic-acid binding proteins (including chromatin-binding proteins containing chromodomains and SET domains) that appear to interact with complexes containing RNA, but whose exact specificity is unknown. Indeed, at least some "transcription factors" have a higher affinity for nucleic acid structures involving RNA than for double-stranded DNA.

In addition, a significant proportion of the human genome appears to be under evolutionary selection, including thousands of ultra-conserved noncoding sequences and transposon-free regions that have remained unchanged throughout mammalian evolution, suggesting extended regions of complex regulatory information that operate via unknown mechanisms, observations that are hard to reconcile with current models of gene regulation. Many noncoding regions are conserved between species in complex patterns that are not evident from pairwise comparisons alone, suggesting that many sequences are under negative or positive selection in different lineages, presumably related to their common ontogeny and phenotypic differences driven by adaptive radiation, respectively.

Such considerations suggest that noncoding RNAs derived from introns and the exons of noncoding RNA genes are not evolutionary debris or transcriptional noise, but rather have evolved to produce functional signals in parallel with protein-coding sequences that enable cross talk between genes (efference signals or eRNAs), and comprise a highly interconnected regulatory network. Indeed, at least some introns are processed to stable RNAs and trafficked to different subcellular locations. Many microRNAs and all snoRNAs are derived from introns, and those that are known are likely to represent just the tip of a very large iceberg. What was dismissed as junk because it was not understood may well encode a critically important hidden layer of genetic programming.

If this is correct, the higher eukaryotes have developed (and by implication require) a much more advanced regulatory system than prokaryotes. This conclusion is supported by an information theoretic analysis and empirical data that show that regulatory networks are accelerating networks and that bacteria have been likely limited in their complexity by a regulatory system based simply on analogue controls (i.e. proteins): the numbers of regulators in prokaryotes grows quadratically with genome size, and extrapolation of the curve suggests that prokaryotes have reached saturation in terms of the number of regulators, probably 
early in their evolutionary history.

This in turn suggests that eukaryotes must have found a solution to this problem and breached this limit by evolving a new regulatory system, presumably based on sequence-specific RNA signaling. This system would, in principle and in practice, represent a quasi-digital feed-forward control system which sets the trajectories of differentiation and development in concert with analog protein-based sensor and signaling pathways that relay contextual cues to supplement, and to correct stochastic errors in, the endogenous program.

Variation in this regulatory architecture may be equally if not more important than variation in the analogue components of the cell (proteins) in determining the major phenotypic differences between individuals and species, including quantitative trait variation, sensitivity to environmental parameters and susceptibility to disease. Understanding this system and its underlying principles may provide a new framework and heuristic for understanding the programming of the development of plants and animals, with enormous implications for biology, medicine and biotechnology. It may also provide a formal link between network theory and complexity theory, and insights into self referential programming and the design parameters of ordered complex systems in general.

\section{Open Lecture Series 1: 100 Ways to Crack the Genome}

OLS- MARLOW, Florence ${ }^{1}$, HOLLOWAY, Beth ${ }^{1}$, WAGNER, 1-1 Daniel ${ }^{1}$, DOSCH, Roland ${ }^{1}$, MEI, Wenyan ${ }^{1}$, GUPTA Tripti $^{1}$, and MULLINS, Mary ${ }^{1}\left({ }^{1}\right.$ University of Pennsylvania School of Medicine, Department of Cell and Developmental Biology, Philadelphia, PA 19104, USA)

Maternal control of vertebrate development: moleculargenetics from the zebrafish

To understand the processes controlled by maternal factors in vertebrates and identify and study key genes involved, we are performing a maternal-effect mutant screen in the zebrafish. We have identified a large number of maternal-effect mutants with specific defects in egg development, egg activation, blastodisc formation, egg and embryonic polarity, cell cleavage, the midblastula transition, embryonic morphogenesis, and body plan formation. Most mutants exhibit unique phenotypes, not previously observed in zygotic mutants, indicating the specific maternal controls in vertebrate development. Positional cloning of the mutated genes is underway, with two mutant genes already identified. Molecular-genetic studies of a subset of the mutants, including an animal-vegetal polarity, a morphogenesis and a dorsal-ventral axis formation mutant will be discussed.

OLS- UEDA, Hiroki R. ${ }^{1}$ ( ${ }^{1}$ Laboratory for Systems Biology, 1-2 Center for Developmental Biology, RIKEN, Kobe, Japan)

System-level Identification of Mammalian Circadian Clocks

Mammalian circadian clocks consist of complexly integrated regulatory loops, making them difficult to elucidate without both the accurate measurement of system dynamics and the comprehensive identification of network circuits. Toward a system-level understanding of this transcriptional circuitry, we identified clock-controlled elements on 16 clock and clock-controlled genes in a comprehensive surveillance of evolutionarily-conserved ciselements and measurement of their transcriptional dynamics using an in vitro validation system ${ }^{\mathbf{1}}$, in which cultured fibroblasts transiently transfected with clock-controlled reporter vectors exhibited robust circadian bioluminescence.

Here we demonstrate the roles of E-boxes/E'-boxes, DBP/ E4BP4 binding elements (D-boxes) and RevErbA/ROR binding elements(RREs) on nine, seven and six genes, respectively. Our results indicate that circadian transcriptional circuits are governed by two design principles: E-box/E'-box and RRE regulation follows a "repressor precedes activator" pattern, resulting in delayed transcriptional activity, while D-box regulation exhibits a "repressor antiphasic to activator" mechanism, which generates high-amplitude transcriptional activity. Our analysis further suggests that E-box/E'-box regulation represents a topological vulnerability in mammalian circadian clocks, which has been functionally verified using in vitro phenotype assay systems ${ }^{2}$

In this conference, we will report a current progress in systemlevel identification of mammalian circadian clocks, and also present a development of "molecular-timetable" methods for detection of body time and rhythm disorders ${ }^{3}$.

1. Hiroki R. Ueda et al. "A transcription factor response element for gene expression during circadian night" Nature, 418, 534539, 2002

2. Hiroki R. Ueda et al. "System-level Identification of Transcriptional Circuits Underlying Mammalian Circadian Clocks" Nature Genetics, 37, 187-192, 2005.

3. Hiroki R. Ueda et al. "Molecular-Timetable Methods for Detection of Body Time and Rhythm Disorders from Singletime-point Genome-wide Expression Profiles" PNAS, 101, 11227-11232, 2004. 